\title{
Brote por pseudomonas aeruginosa, en el área de atención ambulatoria de heridas quirúrgicas, en pacientes posmastectomizadas
}

\author{
Diana Vilar-C ompte, MC, MSc, (1) Benedicte Jacquemin, MC, ${ }^{(1)}$ Adriana Díaz-G onzález, Q FB, ${ }^{(2)}$ \\ Consuelo Velásquez, Q FB, ${ }^{(2)}$ Patricia Volkow, MC. .1) $^{(1)}$
}

\section{Vilar-Compte D, Jacquemin B, Díaz-González A, Velásquez C, Volkow P. Brote por Pseudomonas aeruginosa, en el área de atención ambulatoria de heridas quirúrgicas, en pacientes posmastectomizadas. Salud Publica Mex 2003;45:371-378. El texto completo en inglés de este artículo está disponible en: http://www.insp.mx/salud/index.html}

\section{Resumen}

Objetivo. D escribir un brote por Pseudomonas aeruginosa, en el área de atención ambulatoria de herida quirúrgica, en pacientes posmastectomizadas. Material y métodos. Entre el 13 de marzo y el 18 de mayo de 2000 se definió como caso a las pacientes del Instituto $\mathrm{N}$ acional de Cancerología de la Ciudad de México, mastectomizadas con infección quirúrgica por $\mathrm{P}$ aeruginosa resistente a ciprofloxacina y gentamicina. Se to maron cultivos de los antisépticos, del material de curaciones, del agua y del personal de salud. Se efectuó un análisis de casos y controles. Resultados. Se identificaron 13 infecciones tardías del sitio quirúrgico por P aeruginosa. La Pseudomona se aisló de las narinas de la enfermera y de las gasas que ella misma colocaba sobre la mesa de mayo, pero no de los paquetes de gasas cerrados del área de curaciones ambulatoria de tumores mamarios. El 14 de abril de 2000 se transfirió a la enfermera a otra área y se instauraron medidas estrictas para el control de infecciones. D espués de esta fecha se diagnosticar on cuatro casos más. La radioterapia fue el único factor de riesgo asociado con la infección (RM=5.1, IC 95\%=1.1-28.4). Conclusiones. El brote probablemente estuvo causado en

\author{
Vilar-Compte D, Jacquemin B, Díaz-González A, \\ Velásquez C, Volkow P. \\ Outbreak of postmastectomy \\ wound infections caused by Pseudomonas aeruginosa \\ in an ambulatory surgical care setting. \\ Salud Publica Mex 2003;45:371-378. \\ The English version of this paper \\ is available at: http://www.insp.mx/salud/index.html
}

Este trabajo fue presentado en elVeinticinco C ongreso A nual de laA sociación Mexicana de Infectología y Microbiología C línica, en León, G uanajuato, del 28 de junio al 1 de julio de 2000.

(1) Departamento de Infectología. Instituto $N$ acional de Cancerología, México, D F, México.

(2) Laboratorio Clínico. Instituto $\mathrm{N}$ acional de Cancerología. México, D F, México.

Fecha de recibido: 5 de agosto de 2002 - Fecha de aprobado: 19 de mayo de 2003

Solicitud de sobretiros: Dra. DianaVilar Compte, D epartamento de Infectología, Instituto N acional de Cancerología. Avenida San Fernando 22, colonia Sección XVI Tlalpan, 14080 México, DF, México.

Correo electrónico: diana_vilar@yahoo.com.mx 
un inicio por una fuente común y se extendió por infección cruzada entre las pacientes. Las prácticas deficientes detectadas en el manejo de heridas y drenajes condujo a instaurar medidas preventivas específicas. El texto completo en inglés de este artículo está disponible en: http:// www.insp. $\mathrm{mx} / \mathrm{salud} / \mathrm{index}$.html

Palabras clave: infecciones de sitio quirúrgico; P aeruginosa; brote epidémico; México
The poor compliance with infection control practices during wound cleaning and drainage led to implementing a series of specific preventive interventions. The English version of this paper is available at:http://w ww.insp.mx/salud/index.html

Key words: surgical wound infections; P seudomonas aeruginosa; disease outbreaks; Mexico
$\mathrm{L}$ a Pseudomonas aeruginosa es un bacilo Gram negativo no fermentador de la glucosa, capaz de permanecer por tiempos prolongados en líquidos y superficies como antisépticos, alimentación parenteral, equipo de inhaloterapia, fluidos de diálisis y grifos de agua, entre otros. En contraste, es excepcional encontrarla como parte de la microflora normal de los individuos sanos, en quienes se ha aislado en 0 a $6.6 \%$ en axilas, periné, tracto respiratorio y faringe, y en 2.6 a $24 \%$ en heces. ${ }^{1-3}$

La P aeruginosa es uno de los patógenos nosocomiales más frecuentes. ${ }^{4}$ En nuestra institución ha ocupado el tercer sitio en frecuencia dentro de los aislamientos intrahospitalarios en los últimos cinco años. * En las unidades de cuidados intensivos es frecuente en casos de bacteremias y de neumonías, particularmente en los pacientes intubados, en quienes, además, suele ser multirresistente e incrementa la mortalidad asociada. ${ }^{5,6}$

Los brotes por Pseudomonas representan 5\% de las infecciones nosocomiales. La especie que más comúnmente se ha aislado es la $\mathrm{P}$ aeruginosa y se ha asociado con la contaminación de fuentes comunes como agua, antisépticos y equipo médico, como broncoscopios. $^{7}$

Los brotes por P aeruginosa en cirugía limpia son inusuales, al igual que la transmisión de este germen por personal colonizado, ${ }^{8,9}$ más aún, no se ha descrito antes en una unidad ambulatoria de cuidados posquirúrgicos de heridas. En este trabajo se describe un brote por $P$ aeruginosa en infecciones de la herida quirúrgica, en pacientes posmastectomizadas, en el Instituto Nacional de Cancerología de la Ciudad de México, en donde la fuente probable de infección fue la enfermera encargada del área de curaciones ambulatorias del Departamento de Tumores Mamarios (TM), la cual era portadora de $\mathrm{P}$ aeruginosa en las narinas.

\footnotetext{
* Instituto Nacional de Cancerología. Informe Anual del Comité de Control de Infecciones. 1993-1999. Documento interno.
}

\section{Material y métodos}

\section{Descripción del brote}

El Instituto Nacional de Cancerología (INCan) es un hospital de enseñanza; tiene 150 camas para pacientes con cáncer. El Departamento de TM realiza alrededor de 45 cirugías por mes, en su mayoría mastectomías radicales, las que se consideran cirugías limpias. En 1993 el Departamento de Infectología inició un programa prospectivo, de vigilancia por 30 días, de todas las cirugías efectuadas en el hospital. ${ }^{10}$ En los años previos a 2000 la frecuencia de aislamientos por P aeruginosa en el Departamento de TM fue de 9.1\%, $10.3 \%$ y $18 \%$ para 1997, 1998 y 1999, respectivamente. De acuerdo con los datos del Programa de Vigilancia de Cirugías del INCan los aislamientos más frecuentes en ese servicio fueron $\mathrm{E}$ coli, estafilococos y otras enterobacterias.* $^{*}$

Entre el 13 de marzo y el 18 de mayo de 2000 se observó un incremento inesperado en las infecciones del sitio quirúrgico (ISQ), ocasionadas por P aeruginosa en cirugía radical de mama. Todas las infecciones fueron diagnosticadas tardíamente ( $>=7$ días de la cirugía), y se identificaron después de la primera visita posoperatoria ambulatoria. En esta visita se les hacía limpieza de la herida y del drenaje a las pacientes. Todas las cepas de P aeruginosa presentaron el mismo patrón de resistencia, el cual era diferente al de resistencia habitual de P aeruginosa en nuestra institución.

\section{Estudio epidemiológico y de laboratorio}

Se definió ISQ de acuerdo con los criterios del Centro para el Control de las Enfermedades, de los Estados Unidos de América. ${ }^{11}$

Se definió como caso a toda paciente con mastectomía radical realizada en el INCan y con ISQ por P aeruginosa resistente a gentamicina y ciprofloxacina (Gm-CPO-R), sólo sensible a ceftazidima y carbapenems (CAZ, carbap-S). Se parearon (por frecuencia) 
dos controles para cada caso de ISQ por $\mathrm{P}$ aeruginosa Gm-CPO-R, de acuerdo con el tipo de procedimiento y la fecha de la cirugía ( \pm 4 días). Los controles fueron pacientes mastectomizadas en el INCan, seguidas por un mínimo de 30 días sin diagnóstico de ISQ.

Se recolectaron variables demográficas, quirúrgicas y del posoperatorio, como manejo del drenaje, uso de antibióticos y el número de curaciones posoperatorias efectuadas en forma ambulatoria (que incluyeron limpieza de la herida con agua y antisépticos, así como irrigación con agua corriente y limpieza del drenaje) antes y después del diagnóstico de ISQ (cuadro I).

Al detectar el brote (29 marzo de 2000) se tomaron cultivos ambientales, de los antisépticos, del agua empleada para irrigación y limpieza del drenaje, del lavabo, y de todo el material utilizado para efectuar la curación de la herida y el drenaje, que incluía gasas, tijeras, pinzas, apósitos y palillos de madera para purgar el drenaje. Posteriormente, se tomaron muestras para cultivo de narinas, manos y faringe del personal que laboraba en el cubículo de curaciones ambulatorias.

\section{Cuadro I}

Características de las pacientes. Análisis bivariado. México, 2000

\begin{tabular}{|c|c|c|c|}
\hline Variable & Casos & Controles & RM (IC 95\%) \\
\hline Edad* & $52.4 \pm 12.7$ & $46.4 \pm 8.9$ & \\
\hline Tabaquismo $0^{\ddagger}$ & $3(23.1)$ & $4(15.4)$ & $1.6(0.2-11.7)$ \\
\hline Diabetes $^{\ddagger}$ & $1(7.7)$ & $4(15.4)$ & $0.6(0.1-9.0)$ \\
\hline Hipertensión $n^{\ddagger}$ & $3(23.1)$ & $6(23.1)$ & 1.0 \\
\hline Radioterapia preoperatoria ${ }^{\ddagger}$ & $9(69.2)$ & $8(30.7)$ & $5.1(1.1-28.4)^{\S}$ \\
\hline $\begin{array}{l}\text { Q uimioterapia } 30 \text { días antes } \\
\text { de la cirugía }{ }^{*}\end{array}$ & $1(7.7)$ & $4(15.4)$ & $0.5(0.08-5.4)$ \\
\hline Infección a distancia ${ }^{\ddagger}$ & $3(23.1)$ & $4(15.4)$ & $1.6(0.2-11.7)$ \\
\hline Tiempo quirúrgico $(\mathrm{min})^{*}$ & $145 \pm 38.5$ & $128.1 \pm 46.7$ & \\
\hline Leucocitos* & $5092 \pm 1895$ & $5340 \pm 2510$ & \\
\hline Antibióticos profilácticos ${ }^{\ddagger}$ & $11(84.6)$ & $15(57.7)$ & $3.6(0.5-39.7)$ \\
\hline $\begin{array}{l}\text { Presencia del drenaje } \\
\text { al momento de la infección } \\
\text { del sitio quirúrgico }\end{array}$ & $10(77.7)$ & $14(53.8)$ & $2.8(0.5-19.4)$ \\
\hline Días de drenaje* & $16.8 \pm 5.5$ & $13.5 \pm 5.8$ & \\
\hline $\begin{array}{l}\text { Número de curaciones* } \\
\text { * Media } \pm \text { desviación estánda } \\
\text { ‡ N úmero y porcentaje } \\
\S=0.05\end{array}$ & $5.5 \pm 2.6$ & $4.8 \pm 2.0$ & \\
\hline
\end{tabular}

\section{Microbiología}

Las muestras para cultivo se obtuvieron por aspiración de la secreción sospechosa con una jeringa o con un hisopo estéril (culturete) y se sembraron en agar sangre, MacConkey y chocolate. Para los cultivos atmosféricos se dejaron abiertas por 10 minutos las cajas de Petri con el medio de cultivo, repartidas en cuatro puntos del cuarto de curaciones. Para las superficies y el lavabo se mojó con agua estéril la punta de un hisopo, y se efectuó un rastreo de la superficie. Para el cultivo del agua y de las soluciones empleadas para la curación se tomaron $5 \mathrm{ml}$ del líquido, y se siguió el protocolo establecido para la siembra y cultivo de orina. Los cultivos de muestras de manos, faringe y narinas del personal se tomaron de manera convencional, sin previo aviso a las personas estudiadas.

La identificación de las cepas de $P$ aeruginosa se realizó a través del Micro Scan (AutoScan4. Dade Behring). Para cada una de las cepas se montó una prueba de difusión de disco (Kirby-Bauer), de acuerdo con los estándares del NCCLS, para corroborar los resultados obtenidos por el Micro Scan.

\section{A nálisis de casos y controles}

Se efectuó un análisis de casos y controles para identificar los factores de riesgo. De acuerdo con el tipo de variable y su distribución se efectuó la prueba t de Student o la de U de Mann-Whitney. Para cuantificar la asociación de la ISQ con los posibles factores de riesgo se calcularon la razón de productos cruzados (RM) e intervalos de confianza de 95\% (IC 95\%) mediante el paquete estadístico Epistat 5.0. Se consideró estadísticamente significativa una $\mathrm{p} \leq 0.05$.

\section{Resultados}

Entre el periodo de estudio se identificaron 13 casos de ISQ causados por una P aeruginosa Gm-CPO-R, sólo CAZ-carbap-S. La tasa global de ISQ para la cirugía de mama fue de $29.7 \%$, y de $15 \%$ para las infecciones causadas por $\mathrm{P}$ aeruginosa. La fecha de diagnóstico de cada paciente infectada por P aeruginosa, así como las más relevantes en el estudio del brote, se muestran en la figura 1.

La edad promedio de los 13 casos fue de $52.5 \pm 1.27$ años. Todas las pacientes fueron operadas por el mismo equipo y vigiladas de acuerdo con el protocolo de manejo establecido en ese entonces, que entre otros requisitos incluía la prescripción rutinaria de cefuroxima en el posoperatorio por 7 a 10 días. En el cuadro I se describen las características de los casos y de los controles. En el 
La enfermera es enviada a otro servicio. Se esteriliza todo el material empleado para las curaciones Se vuelve obligatorio el uso de guantes estériles, bata y cubrebocas para las curaciones

Se inician las investigaciones

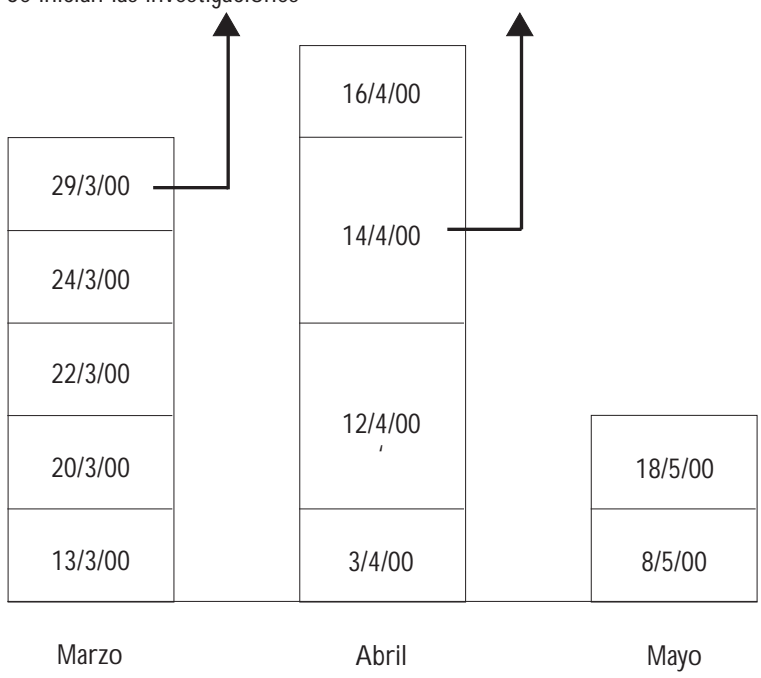

Las fechas mostradas en los recuadros son los días en los que se obtuvo un cultivo positivo a $\mathrm{P}$ aeruginosa multirresistente de las heridas de cada una de las pacientes

Figura 1. Pacientes con infección del Sitio QuirúRgico causada por P aeruginosa. México, Marzo-Mayo 2000

análisis de casos y controles la única variable asociada con ISQ fue la radioterapia previa (RM= 5.1, IC 95\%=1.128.4). El tiempo quirúrgico y el manejo de los drenajes no se asociaron con infección (cuadro I ). La P aeruginosa responsable del brote se aisló de las narinas de la enfermera de la sala de curaciones, así como de las gasas que ella misma colocaba sobre las mesas de Mayo para la curación. Los cultivos tomados directamente de los paquetes cerrados de gasas fueron negativos. Es relevante señalar que ocho de las 13 pacientes infectadas se curaron en el periodo de tiempo en el que la enfermera colonizada con P aeruginosa Gm-CPO-R se encargaba de almacenar y distribuir todo el material de curación, y ayudaba a los cirujanos en la limpieza de la herida y del drenaje. Los cinco casos restantes se diagnosticaron posteriormente a la fecha en la que se transfirió a la enfermera a otra área (14 de abril); sin embargo, las pacientes fueron vistas concomitantemente, y de forma simultánea, con al menos una de las primeras ocho infectadas.
Los cultivos ambientales, de los antisépticos, del agua, de las superficies y de las muestras de manos y narinas del personal fueron negativos a la Pseudomonas, y crecieron solamente otros gérmenes habituales de la flora normal.

Al evaluar la secuencia de los procedimientos realizados por los médicos y enfermeras durante el estudio del brote se identificaron diversas anomalías en el Departamento de TM en el proceso de la curación de las heridas de las pacientes operadas. Además, todo el material empleado para las curaciones, incluyendo los guantes, no era estéril. Ni los médicos ni las enfermeras usaban batas limpias, mascarillas o guantes en forma regular; y se revisaban tres o cuatro pacientes a la vez en un cuarto de aproximadamente $20 \mathrm{~m}^{2}$ en el cual no existía ventilación ni una separación entre las mesas de exploración. Como una práctica común, se desconectaban los tubos del drenaje, y se lavaban con agua corriente de la llave del único lavabo disponible en la sala de curaciones. Posteriormente, los tubos se secaban con toallas de papel no estéril y se recolocaban a la paciente. Un médico podía curar, simultáneamente, a dos enfermas, sin cambio de guantes entre una y otra.

\section{Medidas para el control del brote}

Al identificarse que la cepa de $\mathrm{P}$ aeruginosa, aislada en las narinas de la enfermera del cubículo de curaciones, tenía el mismo patrón de resistencia que la de la $\mathrm{P}$ aeruginosa aislada en las pacientes, la enfermera fue transferida a otra área a partir del 14 de abril de 2000. El seguimiento microbiológico quincenal mostró dos meses después, y luego de un periodo de vacaciones de dos semanas, la negativización de los cultivos de narinas; ello, sin haber recibido tratamiento antimicrobiano alguno.

A partir de la fecha señalada se instauraron medidas estrictas de control de infecciones en el área de curaciones ambulatorias del Departamento de TM y se volvió obligatoria la esterilización de todo el material, el uso de guantes estériles, de bata limpia y cubrebocas para la curación de las heridas, y se estableció que sólo se verían dos pacientes a la vez.

\section{Tratamiento de las pacientes infectadas}

Las pacientes infectadas recibieron diversos tratamientos, de acuerdo con la gravedad y la extensión de la infección (cuadro II). Seis (46.15\%) tuvieron que hospitalizarse para recibir tratamiento intravenoso. Una de ellas, además, requirió debridación quirúrgica. Siete (53.8\%) no recibieron manejo antimicrobiano específico; éstas se manejaron exclusivamente con curaciones diarias de la 


\section{Cuadro II \\ Datos relacionados con la cirugía, el dRenaje, y LA INFECCión DEL SITIO QUiRúrgico Y SU MANEJO. MÉXICO, 2000}

\begin{tabular}{|c|c|c|c|c|c|c|c|}
\hline Caso & $\begin{array}{l}\text { Fecha } \\
\text { de la qx }\end{array}$ & $\begin{array}{c}\text { Fecha del dx } \\
\text { de la infección } \\
\text { del sitio quirúrgico }\end{array}$ & $\begin{array}{l}\text { Días al diagnóstico } \\
\text { de la infección } \\
\text { del sitio quirúrgico }\end{array}$ & $\begin{array}{c}\text { Curaciones previas } \\
\text { al dx de la infección } \\
\text { del sitio quirúrgico } \\
\text { (N) }\end{array}$ & $\begin{array}{l}\text { Curaciones posteriores } \\
\text { al dx de la infección } \\
\text { del sitio quirúrgico } \\
\text { (N) }\end{array}$ & Tratamiento & $\begin{array}{l}\text { Necrosis } \\
\text { del colgajo } \\
(\mathrm{cm})\end{array}$ \\
\hline 1 & 21 feb 00 & 13 mar 00 & 20 & 6 & 12 & CAZ, Q X, Hosp & $7 \times 7$ \\
\hline 2 & 22 feb 00 & 20 mar 00 & 27 & 10 & 3 & CPO & No \\
\hline 3 & 6 mar 00 & 22 mar 00 & 16 & 5 & 14 & & $3 \times 5$ \\
\hline 4 & 6 mar 00 & 24 mar 00 & 18 & 6 & 5 & & No \\
\hline 5 & 14 mar 00 & 29 mar 00 & 15 & 5 & 8 & CAZ, Hosp & $3 \times 5$ \\
\hline 6 & 15 mar 00 & 12 abr 00 & 28 & 9 & 5 & CPO & No \\
\hline 7 & $20 \mathrm{mar} 00$ & 14 abr 00 & 25 & 8 & 2 & & No \\
\hline 8 & 22 mar 00 & 14 abr 00 & 23 & 7 & 6 & & $8 \times 2$ \\
\hline 9 & 22 mar 00 & 12 abr 00 & 20 & 3 & 8 & CAZ, Hosp & No \\
\hline 10 & 27 mar 00 & 3 abr 00 & 7 & 1 & 10 & CPO & No \\
\hline 11 & 4 abr 00 & 16 abr 00 & 12 & 3 & 4 & CAZ, Hosp & No \\
\hline 12 & 17 abr 00 & 8 may 00 & 21 & 4 & 8 & CAZ, Hosp & $7 \times 5$ \\
\hline 13 & 2 may 00 & 18 may 00 & 16 & 6 & 2 & Hosp, CPO & No \\
\hline
\end{tabular}

CAZ $=$ Ceftazidima, $C P O=$ Ciprofloxacina, Hosp $=$ Hospitalización, $Q x=$ Cirugía

El caso 12 desarrolló una osteomielitis del esternón que requirió una nueva hospitalización, antibióticos IV y debridación quirúrgica Los casos 3, 4, 7 y 8 no recibieron antibióticos como parte de su tratamiento. Se manejaron con curaciones y limpiezas continuas

herida y tuvieron buena evolución. En el cuadro II se muestran los datos relacionados con la cirugía, el manejo posoperatorio y el tratamiento de las pacientes infectadas. Se muestra, asimismo, si existió, concomitantemente con la infección, necrosis de los colgajos.

\section{Discusión}

En la literatura se ha informado sobre diversos brotes causados por Pseudomonas. ${ }^{7,8,11-15}$ La mayor parte de éstos son neumonías, asociadas a la estancia en unidades de cuidados intensivos, $3,6,8,12,16,17$ y en un menor porcentaje a bacteremias y a infecciones relacionadas con procedimientos endoscópicos;en un número reducido se han asociado con infecciones del sitio quirúrgico. ${ }^{5,12,13,16-18}$ Este patrón probablemente se encuentra relacionado con la complejidad de los pacientes en este tipo de unidades y con la invasividad a la que son sometidos.

En el brote descrito las pacientes afectadas se encontraban en una situación muy diferente a lo que normalmente ocurre en las unidades de cuidados intensivos; sin embargo, la extensión de la cirugía, la permanencia prolongada de los drenajes durante el posoperatorio, las visitas frecuentes, para el cuidado de la herida y del drenaje, en una sala de curaciones mal ventilada y con hacinamiento, así como las prácticas deficientes en el control de infecciones es probable que hayan facilitado la aparición de este brote por $\mathrm{P}$ aeruginosa, el que incrementó la tasa de ISQ en un servicio de cirugía de mama con tasas de infección quirúrgica mayores a las informadas en la literatura anglosajona $(1.5 \%$ a $25 \%) .{ }^{19-24}$

No es frecuente que el personal del hospital sea un reservorio de $\mathrm{P}$ aeruginosa; sólo el personal que atiende pacientes hospitalizados fuertemente contaminados (por ejemplo, quemados) puede ser colonizado en nariz, faringe o inclusive en la piel hasta en $5 \% .15,25$ Lo anterior evidencia lo inusual del brote descrito, ya que además, se trata de un brote en pacientes posoperadas con infección tardía del sitio quirúrgico, manejadas en un servicio ambulatorio de curaciones.

La $\mathrm{P}$ aeruginosa responsable del brote fue aislada originalmente de las narinas de la enfermera y de las gasas depositadas por ella misma en las mesas de Mayo. Cabe señalar que estas gasas ya se encontraban fuera del paquete, y los cultivos de los paquetes cerrados fueron negativos. Esta contaminación posiblemente ocurrió a través de las manos de la propia enfermera. 
La transmisión de la Pseudomonas a través de las manos del personal de salud se ha postulado como un mecanismo frecuente de transmisión en un brote, pero pocas han sido las ocasiones en las que esto se ha documentado durante el mismo, y tal y como lo mencionan Widmer y colaboradores, ${ }^{8}$ sólo un estudio de cohorte prospectivo, con cultivos repetidos a lo largo del tiempo en pacientes y personal de salud, podría demostrar la adquisición y la transmisión de la P aeruginosa por medio de las manos del personal hospitalario, hecho que muy probablemente ocurrió en la mencionada sala de curaciones. En el brote descrito, todas las infecciones de herida se diagnosticaron después de la primera semana del posoperatorio; 8 de las 13 pacientes infectadas fueron atendidas durante el tiempo en el que la enfermera colonizada almacenaba y distribuía el material de curación; además de que ésta auxiliaba a los cirujanos durante la limpieza de la herida y de los drenajes; los cinco casos restantes no estuvieron relacionados temporalmente con la enfermera, pero sí con al menos una de las primeras ocho pacientes infectadas, lo que sugiere que en este brote también hubo contaminación cruzada.

La contaminación cruzada como ruta de colonización e infección por $\mathrm{P}$ aeruginosa, tanto en un brote epidémico como en condiciones habituales, sugiere un bajo apego a las medidas de control de infecciones, ${ }^{8,17}$ hecho documentado y atendido en forma inmediata después de la detección de este brote. Posteriormente a la modificación de las prácticas en el cuidado de heridas y drenajes, y al cambio de la enfermera colonizada, se controló el brote por Pseudomonas y se inició una serie de medidas preventivas específicas en el Departamento de TM. En el cuadro III se describen las medidas instauradas a lo largo de varios meses.

En el análisis de casos y controles el único factor de riesgo asociado con la infección de la herida en esta serie fue la radioterapia preoperatoria ( $\mathrm{RM}=5.0$, IC $95 \%=1.1-28.4$ ); sin embargo, consideramos que éste no es un factor exclusivo para las infecciones causadas por $\mathrm{P}$ aeruginosa. Es probable que durante el brote las pacientes más vulnerables fueron las que se infectaron.

El papel de la radioterapia preoperatoria en las complicaciones posoperatorias de las mastectomías ha sido pobremente estudiado; sin embargo, la radioterapia incrementa la fibrosis y disminuye la vascularidad, lo que favorece la necrosis tisular, la infección y ulceración de la piel y de los tejidos subyacentes. ${ }^{26}$ Estos fenómenos son comunes en la población operada por cáncer de mama en nuestra institución y, en un estudio de casos y controles en pacientes mastectomi-

\section{Cuadro III \\ Cronología de las medidas de intervención. MÉxico, 2000}

Abril 2000 Esterilización de todo el material de curación. U so obligatorio de cubrebocas, bata y guantes estériles para efectuar las curaciones

Enero 2001 Introducción de un drenaje cerrado parcialmente siliconizado con tubos redondos (Hemovac)

Junio 2001 Suspensión de la prescripción rutinaria de 7-10 días de antibiótico en el posoperatorio. Uso exclusivo de antibióticos profilácticos

Introducción del drenaje Biovac (siliconizado en su totalidad con tubos planos)

Reporte individual a los cirujanos de las complicaciones de la herida

Introducción de mini-equipos estériles para curación

A gosto 2001 Remodelación del área de curaciones. D esaparecen las curaciones simultáneas de las pacientes

zadas ${ }^{27}$ la radioterapia preoperatoria ha mostrado ser un factor de riesgo para las ISQ en general independientemente del patógeno involucrado $(\mathrm{OR}=2.3$, IC $95 \%=1.2-4.3$ ).

La $\mathrm{P}$ aeruginosa se identificó a través del Micro Scan, que ha demostrado identificar correctamente $95.4 \%$ de todas las enterobacterias y $94.2 \%$ de los no fermentadores. ${ }^{28}$ Por medio de la técnica automatizada, y del método de difusión en disco (Kirby-Bauer), todas las $\mathrm{P}$ aeruginosa identificadas fueron sensibles sólo a cefatzidima, imipenem y meropenem. Esta cepa fue resistente a aminoglucósidos y quinolonas, hecho que dificultó su manejo y aumentó los costos de atención durante el tratamiento de estas pacientes, ya que fue necesario hospitalizar a seis de ellas.

La prevalencia de microrganismos multirresistentes es un problema creciente, y en el ámbito intrahospitalario cobra un particular interés..$^{29} \mathrm{La}$ Pseudomonas aeruginosa se considera un microrganismo centinela, y una modificación en el patrón de resistencia es una determinante muy importante en los sistemas de vigilancia epidemiológica, ya que uno de los factores de riesgo asociados a la multirresistencia es el uso expandido, y en ocasiones indiscriminado, de antibióticos en hospitales y en la comunidad. ${ }^{3,6}$

En el brote descrito, la P aeruginosa fue resistente a ciprofloxacina y amikacina, hecho que contrasta con el patrón habitual de nuestra institución, ya que en el periodo de 1998 a 2001 la Pseudomonas aislada en infecciones de herida quirúrgica mostraron una sus- 
ceptibilidad a ciprofloxacina por arriba de $90 \%$ y a aminoglucósidos de $70 \%{ }^{30}$ Estos mismos datos se han corroborado para la Pseudomona provenientes de orina y sangre, ${ }^{*}$ fenómeno inverso a lo informado en países anglosajones, en donde han encontrado un porcentaje de resistencia mayor a quinolonas y a otros antimicrobianos empleados en el tratamiento de las infecciones ocasionadas por este germen. 6,17,29,31

En esta serie no fue posible realizar pruebas de tipificación molecular, que nos hubieran permitido corroborar si la cepa aislada de la enfermera era la misma que la aislada de las pacientes. Actualmente se recomienda contar con algún tipo de análisis molecular cuando ocurre un brote; sin embargo, en instituciones como la nuestra, y en muchas otras del país y del resto de Latinoamérica no se cuenta con esta tecnología. Algunos autores han mencionado que, aunque recomendable, no es la panacea, ${ }^{32}$ y el laboratorio clínico (a través de la identificación y el patrón de sensibilidad antimicrobiana) y la vigilancia cotidiana siguen siendo los elementos clave para la identificación de un brote. En este brote en particular, algunos de los hallazgos por microbiología y epidemiología clásica sugieren que la cepa de $\mathrm{P}$ aeruginosa fue la misma. Elementos que apoyan esta conclusión son el mismo patrón de resistencia, y la rápida caída en las infecciones reportadas, una vez que se retiró a la enfermera de la sala de curaciones de TM. La aparición tardía y más lenta de nuevos casos una vez que la enfermera salió de la sala de curaciones probablemente se explican por la contaminación cruzada entre las pacientes a través de las manos del personal. Después del 18 de mayo de 2000, y de la introducción paulatina de un programa preventivo específico en cirugía mamaria, no se han detectado cepas de $P$ aeruginosa multirresistente, ni nuevos brotes.

Ahora que la atención médica de los enfermos se está canalizando hacia las unidades de atención ambulatoria es indispensable contar con un programa permanente y prospectivo de vigilancia de la herida quirúrgica. En este estudio, gracias al programa de vigilancia de las mastectomías por más de 30 días, lo que incluye el área de curaciones ambulatorias, fue posible detectar la existencia de este brote.

Las condiciones descritas de la práctica médica cotidiana probablemente ocurren en muchos países en desarrollo, en donde el hacinamiento, la falta de lugares de trabajo adecuados y la ausencia de protocolos

\footnotetext{
* Cornejo- Juárez P, Velásquez -Acosta C, Díaz -González A, Volkow -Fernández P. Panorama de resistencias antimicrobianas en un hospital de referencia oncológico en un periodo de seis años. Documento no publicado
}

estándares de manejo pueden incrementar el riesgo de la aparición de brotes como el señalado. Es recomendable la vigilancia de las heridas una vez que el paciente ha egresado del hospital, así como establecer protocolos estrictos de prevención y manejo de heridas.

La presente investigación permitió modificar algunas de las prácticas de los cirujanos del Departamento de TM de nuestro hospital, y desencadenó una serie de intervenciones que, a enero de 2003, han disminuido en más de $50 \%$ las ISQ, y mejorado la calidad de la atención en este grupo de pacientes.

\section{Referencias}

1. Kiska D L, Gilligan PH. Pseudomonas. En: Murray PR, Ed. Manual of clinical microbiology. $7^{\text {th }}$ ed. W ashington, DC:ASM Press; 1999:517.

2. Pollack M. Pseudomonas aeruginosa. En: Mandell GL, Bennett JE, D olin $R, E d$. Principles and practice of infectious diseases. $5^{\text {th }}$ ed. Filadelfia, (PA): Churchill Livingstone, 2000:2310.

3. Corona-N akamura AL, Miranda-N ovales MG, Leaños-Miranda B, Portillo-Gómez L, Hernández-Chávez A,Antro-Rendón J et al.

Epidemiologic study of Pseudomonas aeruginosa in critical patients and reservoirs.Arch Med Res 2001;32:238-242.

4. Centers for Disease Control and Prevention. $N$ ational $N$ osocomial Infections Surveillance (N N IS) report, data summary from 0 ctober 1986-A pril 1996, issued May 1996. Am J Infect Control 1996;24: 380-388.

5. Fagon JY, C hastre J, Hance AJ, Montravers P, N ovara A, G ibert C. $N$ osocomial pneumonia in ventilated patients: $A$ short study evaluating attributable mortality and hospital stay. Am J Med 1993;94:281-288. 6. Harris AD, Smith D, Johnson JA, Bradham D D, Roghmann MC. Risk factors for imipenem-resistant Pseudomonas aeruginosa among hospitalized patients. C lin Infect D is 2002;34:340-345.

7.W endt C, Herwaldt LA. Epidemics: Identification and management. En: Wenzel RP, Ed. Prevention and control of nosocomial infections. 3rd edition. Baltimore (MD):W illiams and W ilkins; 1997:177-213. 8.W idmer AF,W enzel RP, Trilla A, Bale MJ, Jones RN , D oebbeling BN . 0 utbreak of Pseudomonas aeruginosa infections in a surgical intensive care unit: Probable transmission via hands of a health care worker. C lin Infect D is 1993;16:372-376.

9. MC N eil SA, N ordstrom-Lerner L, Malani PN , Zervos M, Kauffman C. 0 utbreak of sternal surgical site infections due to Pseudomonas aeruginosa traced to a scrub nurse with onychomycosis. C lin Infect D is 2001;33:317-323.

10. Vilar-Compte D, Mohar A, Sandoval S, D e la Rosa M, Gordillo P, Volkow P. Surgical site infection at the $\mathrm{N}$ ational $\mathrm{C}$ ancer Institute in Mexico:A case control study. Am J Infect Control 2000;28:14-20. 11. Horan TC, G aynes RP, Martone W J,W illiam RJ, Emory TG . CDC definitions of nosocomial surgical site infections, 1992: A modification of $C D C$ definition of surgical wound infections. Infect Control Hosp Epidemiol 1992;13:606-608.

12. 0 strosky-Zeichner L, Báez-Martínez R, Rangel-Frausto MS, Ponce de León S. Epidemiology of nosocomial outbreaks: 14-year experience at a tertiary-care center. Infect Control Hosp Epidemiol 2000;21:527-529. 13. C limo MW, Pastor A, W ong ES. An outbreak of Pseudomonas aeruginosa related to contaminated urodynamic equipment. Infect Control Hosp Epidemiol 1997;18:509-510. 
14. Rudnick JR, Beck-Sague CM, Anderson RL, Schable B, Miller JM, Jarvis W R. G ram-negative bacteremia in open-heart surgery patients traced to probable tap-water contamination of pressure-monitoring equipment. Infect Control Hosp Epidemiol 1996;17:281-285. 15. Lowbury EJL, Fox J.The epidemiology of infection with Pseudomonas pyocynea in a burns unit. J Hyg 1954;52:403-416. 16. Harris AD, Perencevich E, Roghmann MC, Morris G, Kaye KS, Johnson J. Risk factors for piperacillin-tazobactam-resistant Pseudomonas aeruginosa among hospitalized patients. Antimicrob Agents Chemother 2002;46:854-858.

17. Bergmans DCJJ, Bonten MJM, van Tiel FH, G aillard CA, van der Geest S, W ilting RM et al. C ross-colonisation with Pseudomonas aeruginosa of patients in an intensive care unit. Thorax 1998;53: 1053-1058.

18. Jumaa $P, C$ hattopa B. 0 utbreak of gentamicin, ciprofloxacin-resistant Pseudomonas aeruginosa in an intensive care unit traced to contaminated quivers. J Hosp Infect 1994;28:209-218.

19. Broadwater JR, Edwards MJ, Kuglen C, Horto bagyi GN , A mes FC, Balch CM. Mastectomy following preoperative chemotherapy. Strict operative criteria control operative morbidity.Ann Surg 1991;213: 126-129.

20. $O$ 'Brien W O, Hasselgren PO, Hummel RP, C oith R, Hyams D, Kurtzman $\mathrm{L}$ et al. Comparision of postoperative wound complications and early cancer recurrence between patients undergoing mastectomy with or without immediate breast reconstruction. Am J Surg 1993;166:1-5.

21. Terrell GS, Singer JA. Axillary versus combined axillary and pectoral drainage after modified radical mastectomy. Surg Gynecol 0 bstet 1991;175:437-440.

22. C anavase $G, C$ atturich A,Vecchio C, G ipponi M, Tomei D, Sertoli MR et al. Surgical complications related to peri-operative adjuvant chemotherapy in breast cancer. Results of a prospective, controlled randomized clinical trial. Eur J Surg 0 ncol; 1997;23:10-12.

23. Furey PC, MacG illivary DC, C astiglione CL, Allen L. W ound complications in patients receiving adjuvant chemotherapy after mastectomy and immediate breast reconstruction for breast cancer.J Surg 0 ncol 1994;55:194-197.

24. Lefebvre D, Penel N , Deberles MF, Fournier C. Incidence et facteurs de risqué d' infections du site opératoire en chirurgie carcinologique mammaire. Presse Med 2000;29:1927-1932.

25. Arnow PM, Flaherty JP. N onfermentative gram-negative bacilli. En: Mayhall CG, Ed. Hospital epidemiology and infection control. $2^{\text {nd }}$ edition. Filadelfia (FA): Lippincot W illiams and W ilkins, 1999:431-451.

26. Mathes SJ,Alexander J. Radiation injury. Surg 0 ncol C lin N orth Am 1996;5:809-824

27. Vilar-Compte D, Jacquemin B, Robles-Vidal C, Volkow P. Surgical site infections in breast surgery:A case-control study.Abstracts K-1458, Abstracts of the $41^{\text {st }}$ ICAAC, September and December 2001:434.

28. Rhoden D L, Smith PB, Baker CN , Schable B. AutoSC AN -4 system for identification of gram-negative bacilli. J C lin Microbiol 1985;22:915-918. 29. Fridkin SK, Hill HA, Volkova N, Edwards JR, Lawton RM, G aynes RP et al.Temporal changes in prevalence of antimicrobial resistance in 23 U.S hospitals. Emerg Infect D is 2002;8:697-701.

30. Rodríguez-Alvarez M, Roldán-Marín R, Cornejo-Juárez $P$, Vilar-Compte D. Epidemiología y microbiologia de las infecciones de sitio quirúrgico (ISQ ) en un hospital oncológico (1998-2001). Resumen D-51. XXVII Congreso A nual de la Asociación Mexicana de Infectología y Microbiología C línica. IV Congreso de la Asociación Panamericana de Control de Infecciones y Epidemiología Hospitalaria;2002 N oviembre. 28-30 Cancún, Q R, México.

31. Turnridge J, Bell J, Biedenbach DJ, Jones RN . Pathogen occur rence and antimicrobial resistance trends among urinary tract infection isolates in the A sia-W estern Pacific Region: Report from the SEN TRY antimicrobial surveillance program, 1998-1999. Int J Antimicrob Agents 2002;20:10-17.

32. W eber S, Pfaller MA, Herwaldt LA. Role of molecular epidemiology in infection control. Infect D is Clin N orth Am 1997;11:257-278. 